\title{
A válvula de Heimlich no tratamento do pneumotórax*
}

\author{
Ricardo Beyruti ${ }^{1}$, Letícia Engber Odilon Villiger², J osé Ribas Milanez de Campos ${ }^{1}$, \\ Rodrigo Afonso Silva², Angelo FernandeZ ${ }^{1}$, FÁbio Biscegli Jatene ${ }^{3}$
}

Objetivos: A válvula de Heimlich foi descrita para substituir os sistemas de drenagem sob selo d'água. O Serviço de Cirurgia Torácica do Hospital das Clínicas da Faculdade de Medicina da Universidade de

São Paulo realizou este estudo com o objetivo de avaliar sua eficiência no tratamento do pneumotórax, bem como a facilidade de manipulação, segurança e tolerância pelo paciente. Métodos: Foram analisados 108 pacientes com pneumotórax de diferentes etiologias, de junho/97 a agosto/2000. A maioria (76\%) apresentava pneumotórax classificado radiologicamente como médio ou grande, sendo três deles hipertensivos, dos quais um bilateral. Utilizaram-se para a drenagem da cavidade pleural cateteres plásticos de teflon ou poliuretano tipo pig-tail $10,2 \mathrm{~F}, 14 \mathrm{~F}$ ou retos $11 \mathrm{~F}$

conectados à válvula de Heimlich. Nos pacientes pós-toracotomias, a válvula foi conectada diretamente ao dreno torácico. Resultados: 0 período de permanência da válvula variou de um a

120 dias (mediana de quatro dias). A tolerância ao sistema foi considerada boa por $89 \%$ dos pacientes. Não ocorreram complicações atribuíveis ao sistema. A presença de derrame associado em 20 pacientes $(18,5 \%)$ não impediu o bom funcionamento do sistema. Apenas dois $(1,8 \%)$ pacientes evoluíram com enfisema subcutâneo progressivo, optando-se então por dreno tubular 36F sob selo d'água. Em um paciente $(0,9 \%)$ indicou-se toracotomia para descorticação pulmonar. Vinte pacientes $(18,5 \%)$ foram tratados no ambulatório. Conclusões: A válvula de Heimlich mostrou-se eficiente na resolução do pneumotórax de diferentes etiologias e nas fístulas aéreas pós-operatórias. A manipulação foi mais simples do que as historicamente observadas nos sistemas convencionais. A boa tolerância e segurança referidas pelos pacientes foram fator determinante na precocidade da alta hospitalar e incentivo ao tratamento ambulatorial. (J Pneumol 2002;28(3):115-119)

\section{Heimlich valve in the treatment of pneumothorax}

Objective: Heimlich valve has been described as a substitute for water-sealed drainage systems.

The objective of this study by the Thoracic Surgery Service of the "Hospital das Clínicas",

University of São Paulo Medical Center, is to assess its effectiveness in the treatment of pneumothorax and its ease of operation, safety, and patient toleration. Methods: From J une/97

to August/00, 107 patients with pneumotoraxes of different etiologies were analyzed. The majority $(76 \%)$ were classified radiologically as suffering from moderate or large pneumothorax, three hypertensive pneumothorax, and one was bilateral. The pleural cavity was drained with

teflon or polyurethane plastic pig-tail catheters $(10.2 \mathrm{~F}, 14 \mathrm{~F}$, straight $11 \mathrm{~F}$ connected to the

Heimlich valve or the valve was connected directly to the thoracic drain in post-thoracotomy patients). Results: The valve remained in place for one to 120 days (mean 4 days). Tolerance to the system was good in $89 \%$ of cases. There were no serious complication linked to the system. Associated pleural effusion in 20 patients $(18.5 \%)$ did not prevent the system from functioning well. Only two patients (1.8\%) developed subcutaneous emphysema, and therefore, a 36F Tubular drain under water seal was chosen. Thoracotomy for decortication was indicated in one patient

$(0.9 \%)$. Twenty patients $(18.5 \%)$ were treated on an outpatient basis. Conclusions: The

* Trabalho realizado no Serviço de Cirurgia Torácica do Hospital das Clínicas da Faculdade de Medicina da Universidade de São Paulo (HC-FMUSP), São Paulo, SP.

1. Médico Assistente.

2. Médico Ex-residente.

3. Chefe do Serviço.

J Pneumol 28(3) - mai-jun de 2002
Endereço para correspondência - Ricardo Beyruti, Rua J osé Pepe, 80 - 05652-080 - São Paulo, SP. Tel. (11) 3721-8656; fax (11) 37221235; e-mail: rbeyruti@usp.br

Recebido para publicação em 15/12/01. Aprovado, após revisão, em 16/1/02. 
Heimlich valve confirmed its effectiveness in resolving pneumothorax of different etiologies and in postoperative air leaks. Its operation was simpler than that of conventional systems. The good toleration and safety mentioned by the patients was a determining factor in early hospital discharge and a motive for outpatient treatment.

Descritores - Pneumotorax. Pleura. Tratamento. Drenagem portural.

Key words - Pneumothorax. Pleura. Postural drainage.

\section{INTRODUÇÃO}

Henry Heimlich(1), em 1968, idealizou um dispositivo para substituir os sistemas de drenagem sob selo d'água convencionalmente utilizados, apresentando vantagens, tais como: conferir maior mobilidade ao paciente, não necessitar de pinçamento durante 0 transporte e oferecer maior segurança e facilidade de higienização. Propôs, então, uma válvula, de pequenas dimensões, que permite a passagem de fluido ou ar em uma única direção, evitando o refluxo para a cavidade pleural. Além dessas vantagens, seria de fácil utilização e entendimento pela equipe médica, de enfermagem e, inclusive, pelo próprio paciente. 0 sistema também mantém-se funcionando, independentemente de sua posição ou nível, tornando a drenagem pleural mais confiável (Figura 1).

A incidência de pneumotórax espontâneo tem sido estimada em 8/100.000 e a razão homem/mulher é de $6: 1$; a maioria dos pacientes afetados $(85 \%)$ tem menos de 40 anos de idade. Com a evolução e modernização das unidades de terapia intensiva, das técnicas de suporte ventilatório e monitorização, assim como o desenvolvimento de aparelhos e instrumentos de diagnóstico mais invasivos, percebeu-se 0 aumento de complicações, como o pneumotórax iatrogênico, que caracteristicamente, aparece após tentativas de canulação do acesso venoso central, barotrauma, biópsia transbrônquica ou transtoráci$\mathrm{ca}$, entre outras etiologias. Com o ressurgimento da cirurgia redutora de volume para o enfisema pulmonar, os pacientes permanecem por mais tempo com dois ou mais drenos pleurais decorrentes de fístula aérea, o que os mantém restritos ao leito ou à cadeira devido à falta de praticidade dos frascos sob selo d'água. Decorrentes de todos esses fatores, surgiu então a necessidade de formas mais práticas, mais econômicas, eficientes e seguras para 0 manejo desses pacientes ${ }^{(2)}$.

Este estudo tem como objetivo avaliar a eficiência da válvula de Heimlich no tratamento do pneumotórax de diversas etiologias, bem como a facilidade de manipulação, segurança e sua tolerância pelo paciente.
Abreviatura utilizada neste trabalho

CTVA - Cirurgia torácica videoassistida

\section{Casuística e mÉTOdo}

Foram analisados pacientes nos seguintes hospitais: H ospital das Clínicas da Universidade de São Paulo (ICHCINCOR), Hospital Israelita Albert Einstein, Hospital e Maternidade São Luiz e Hospital Sírio Libanês. No período de junho/97 a agosto/2000, 108 pacientes foram tratados, sendo $71(61,2 \%)$ do sexo masculino e $37(38,8 \%)$ do feminino, com idade variando de duas horas de vida a 97 anos (mediana de 53 anos).

A válvula de Heimlich foi utilizada em duas situações distintas: em pacientes com drenos pleurais pós-toracotomias, substituindo os tradicionais frascos sob selo d'água, sendo conectada ao dreno tubular ou então a cateteres de menor calibre do tipo pig-tail 10,2F ou 14F; ou retos $11 F$, instalados por punção e sob anestesia local (conjuntos de drenagem pleural Cook e Arrow) (Figura 2).

Quanto à etiologia, o pneumotórax foi classificado como: 38 (35\%) espontâneos; 25 (23,3\%) por acidente de punção venosa central; $17(15,7 \%)$ pós-toracotomias com ou sem fístula aérea; oito $(7,4 \%)$ por barotrauma decorrente de ventilação mecânica; nove $(8,4 \%)$ pós-procedimentos diagnósticos transtorácicos ou transbrônquicos; seis $(5,5 \%)$ pós-trauma torácico; cinco $(4,7 \%)$ por tocotraumatismo (Tabela 1).

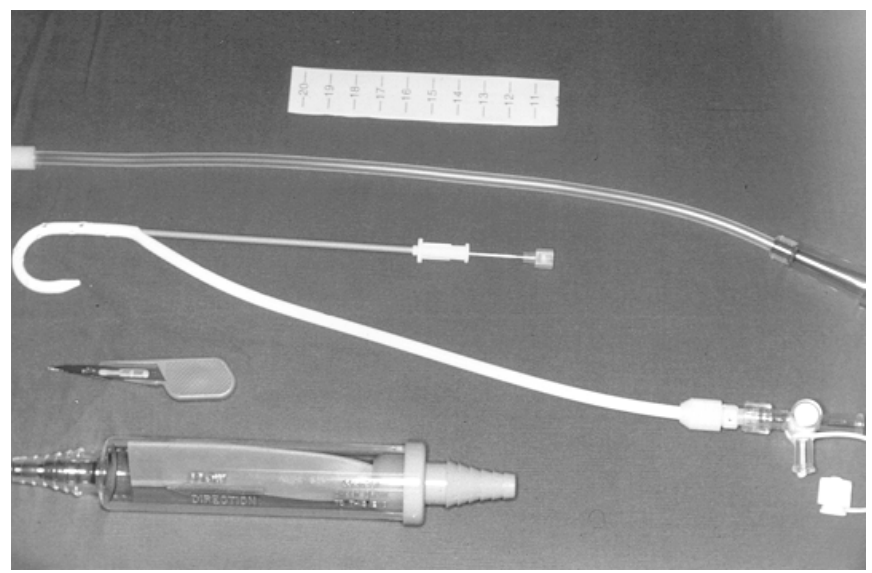

Figura 1 - Conjunto utilizado para a drenagem do pneumotórax, constituído por cateter tipo pigtail com mandril para sua introdução, tubo conector e a válvula de Hemlich 


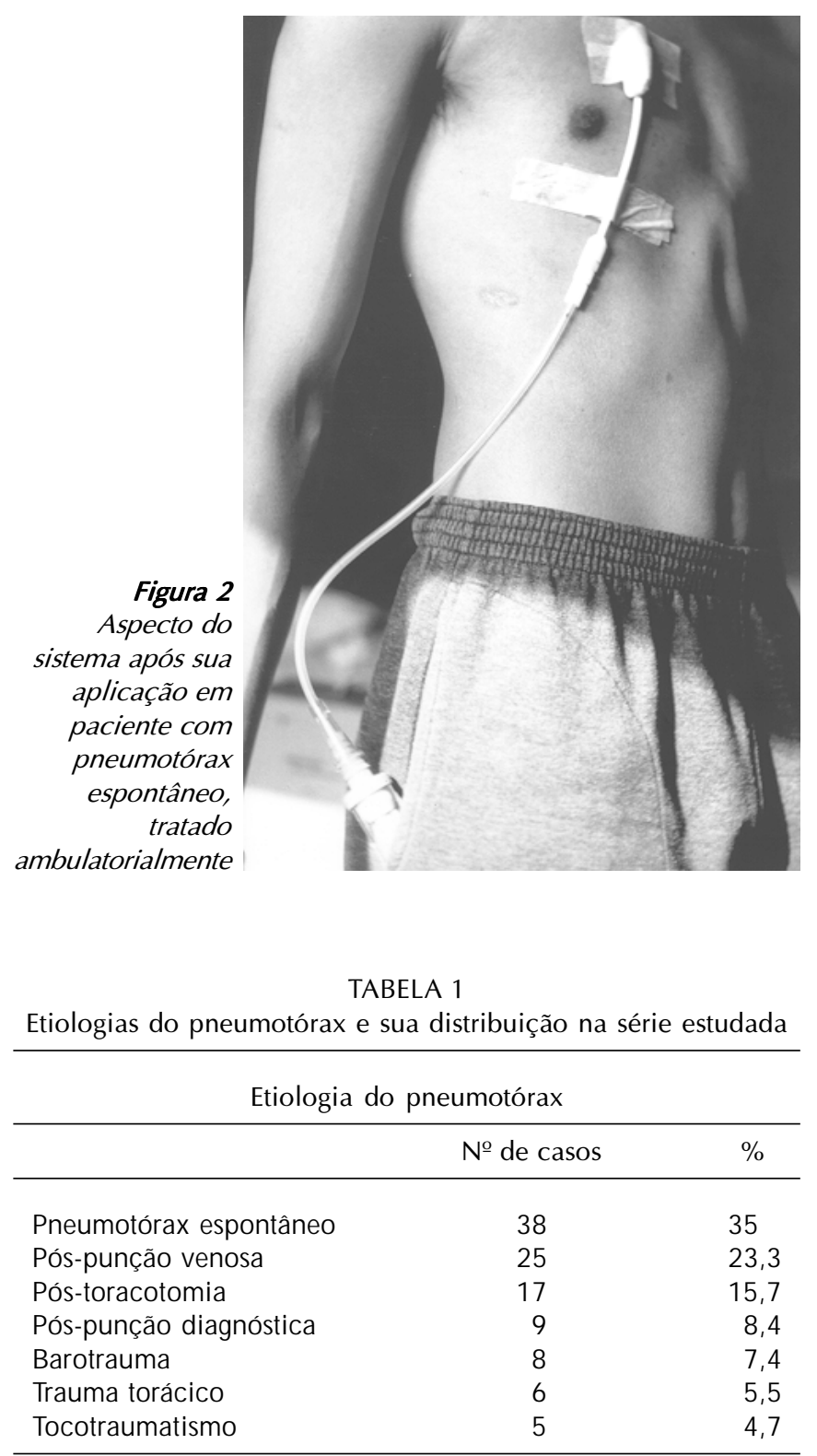

\section{Resultados}

A expansão pulmonar completa foi observada em 103 $(95,37 \%)$ dos 108 pacientes. Observou-se em 83 (80\%) a expansão pulmonar completa nas primeiras 48 horas, quatro $(4 \%)$ no terceiro dia e quatro $(4 \%)$ no quarto dia. Do total, 12 (12\%) pacientes necessitaram de um período maior do que uma semana para a expansão pulmonar completa ocorrer (Figura 3). Dos $42(38,9 \%)$ que apresentaram fístula aérea, 40 (95,3\%) evoluíram com o fechamento das mesmas. 0 tempo de duração das fístulas assim como a permanência da válvula variou de um a 120 dias, com mediana de quatro dias. Um paciente evo-
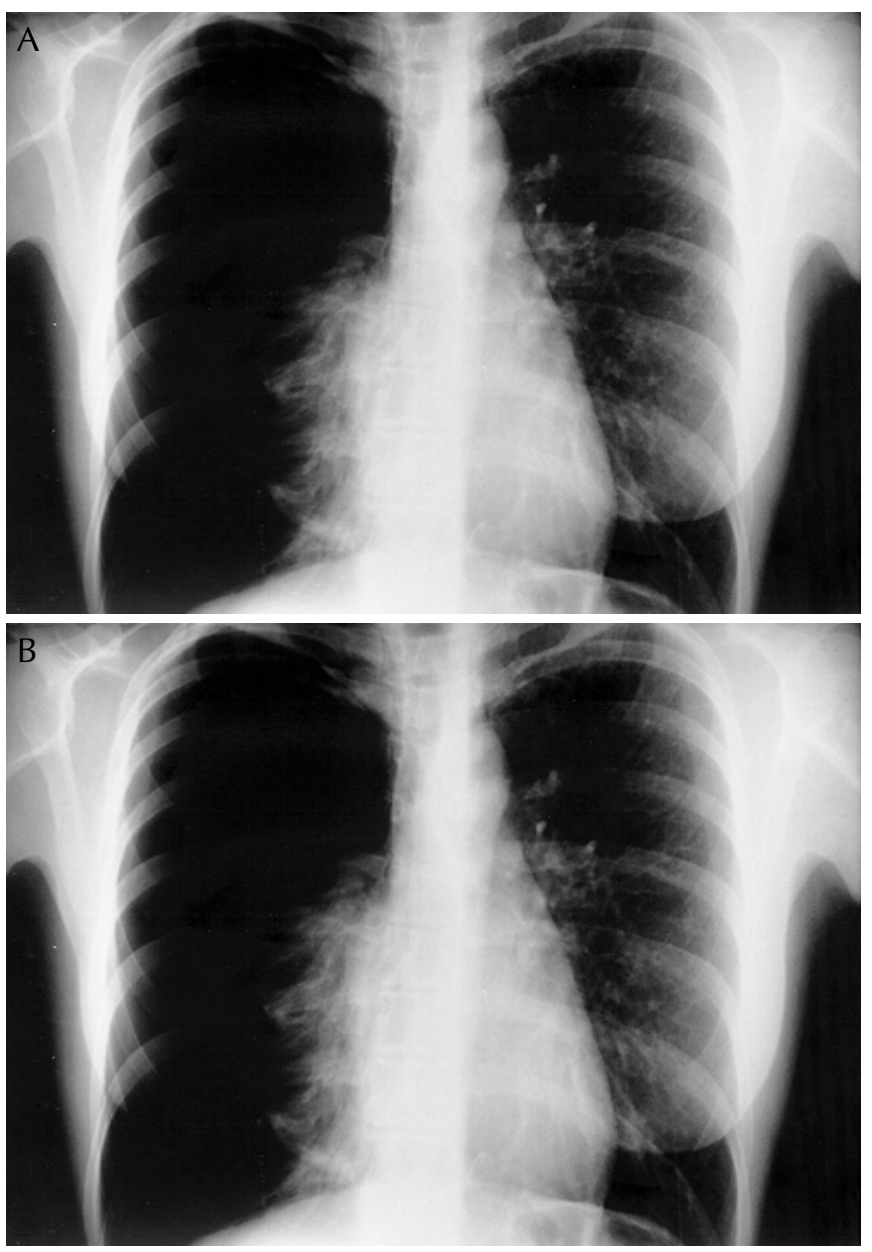

Figura 3 - A) Radiografia de tórax evidenciando colapso do pulmão direito por pneumotórax espontâneo. B) Radiografia de tórax do mesmo paciente, logo após inserção do cateter, com expansão pulmonar.

luiu, depois da retirada do conjunto, com pequeno pneumotórax residual em ápice que se resolveu após uma semana. Um paciente apresentava expansão pulmonar incompleta com presença de bolhas subpleurais em segmento apical, sendo submetido à segmentectomia por cirurgia torácica videoassistida (CTVA).

Derrame pleural associado foi observado em 20 (18,5\%) pacientes, com características que variaram entre amarelo-citrino $12(60 \%)$, sero-hemático sete $(35 \%)$ e hemorrágico um $(5 \%)$, que foram também resolvidos após tratamento. Em um caso foram detectadas células neoplásicas bem como bolhas subpleurais em segmento apical, sendo indicada a segmentectomia apical e pleurodese por CTVA. Um caso apresentava pneumonia com pneumotórax associado e empiema em fase inicial, que evoluiu para encarceramento pulmonar necessitando de descorticação. Um paciente evoluiu, após a retirada do conjunto, com 
pequeno derrame pleural que se resolveu após uma semana.

De 76 pacientes ( $70,4 \%$ do total) que estavam acordados e contactuantes quando da utilização do sistema, 72 deles $(94,8 \%)$ referiram a tolerância como boa, três $(3,9 \%)$ como regular e apenas um $(1,3 \%)$ como ruim. Não foram interrogados os demais $32(29,6 \%)$ por estar sob ventilação assistida, sedados ou inconscientes, sendo que cinco eram neonatos.

Do total de pacientes, os sintomas mais referidos, avaliados previamente à drenagem, foram: dispnéia com 72 citações, sendo o sintoma mais comum, seguido pela dor torácica com 40 citações e a tosse com 14 citações.

A duração da instalação do sistema variou de um a 30 minutos, com média de 15 minutos e 30 segundos. Não houve mortalidade relacionada ao uso da válvula de Heimlich.

Como complicações observou-se a expansão pulmonar incompleta em dois doentes e enfisema de subcutâneo em três outros. Os tratamentos instituídos para tais complicações foram: videocirurgia com bolhectomia apical e pleurodese para os casos de expansão incompleta associada à permanência de fístula com bolhas subpleurais vistas à tomografia de tórax. Nos doentes com enfisema, optou-se pela troca dos cateteres mais finos por drenos tubulares $36 \mathrm{~F}$ com drenagem sob selo d'água, ou a instalação de pressão negativa regulada.

\section{DISCUSSÃO}

0 acúmulo de ar intrapleural é uma afecção que, apesar de simples, tem motivado a publicação de artigos e estimulado o desenvolvimento de vários métodos de tratamento. Existem ainda muitas divergências de opiniões acerca de quando e como tratá-lo $0^{(3,4)}$.

0 conceito de drenagem pleural fechada foi descrito por Playfair, em $1875^{(5)}$, na Inglaterra. Em 1910, Robinson $^{(6)}$ introduziu a aspiração do sistema usando vácuo, mas seu método só foi difundido e ganhou importância mundial após o período de 1918, por ocasião da Primeira Guerra Mundial, quando Evarts Grahan reduziu a mortalidade do empiema de 30 para $3 \%$. Lilienthal(7) descreveu um método simples: um par de frascos coletores e 0 uso do selo d'água no período pós-operatório, em 1926. Estes dados foram confirmados por Brum, em 1929, e finalmente adotado como procedimento de rotina em cirurgia torácica.

Com o intuito de prevenir o refluxo de ar ou fluidos para a cavidade pleural, os drenos torácicos têm sido então conectados usualmente a frascos sob selo d'água, o que muitas vezes confina o paciente ao leito ou à cadeira, dificultando a deambulação. Os frascos devem ser mantidos em nível mais baixo que o tórax do paciente, além do inconveniente da possibilidade de descontinuidade das conexões entre o tubo e o frasco, queda e vazamento da água, o que pode ocasionar um pneumotórax rapidamente progressivo. Além disso, as equipes médica e de enfermagem freqüentemente ficam confusas com os sistemas de tubos e frascos; também a limpeza e esterilização dos sistemas consomem tempo, conforme relatos e observações desde a época do próprio Heimlich(1).

Como mencionado na introdução, a incidência das várias etiologias do pneumotórax tem aumentado recentemente e o desconforto dos sistemas de drenagem sob selo d'água acabaram por determinar a necessidade de um processo alternativo que possibilita maior mobilidade dos pacientes ${ }^{(8-11)}$. A drenagem sob selo d'água funciona como uma válvula que permite a passagem de fluido ou ar em apenas uma direção. A válvula de Heimlich segue o mesmo princípio, valendo-se de duas membranas de borracha; tem como vantagens funcionar em qualquer posição, não correndo o risco de derramar, vazar, refluir, formar bolhas que obstruam o sistema, além de não precisar ser trocada diariamente. Todas elas reduzem consideravelmente a necessidade de pinçamento ou a possibilidade de obstrução do sistema. Se desejado, a válvula também pode ser conectada à sucção regulada. Apesar de o controle pós-operatório do volume ou intensidade da fístula aérea não ser satisfatório, não foram relatadas obstruções ou complicações nas séries estudadas.

A partir de junho/97, com a introdução desse dispositivo em nosso meio, a drenagem pleural em adultos passou a ser realizada também por essa técnica, com bons resultados, principalmente em pacientes com pneumotórax de diferentes etiologias. Durante a realização deste trabalho, aventou-se a possibilidade de utilização da válvula conectada ao sistema de drenagem também em neonatos. Em virtude da escassez de estudos na população pediátrica, o pneumotórax nessa faixa etária é tratado seguindo o mesmo algoritmo estabelecido para os adultos ${ }^{(12)}$, isto é, drenagem pleural com dreno tubular multiperfurado e conexão a um sistema sob selo d'água. Apesar de não encontrar na literatura relatos da conexão da válvula de Heimlich aos sistemas de drenagem para tratamento de pneumotórax em neonatos, apresentamos neste trabalho cinco casos que tiveram boa evolução.

A resolução do pneumotórax com agulhas ou tubos de drenagem convencionais ocorre, em média, dentro de dois ou três dias ${ }^{(3)}$, média também encontrada em nosso estudo. McKenna et al.(13) relataram que a média de permanência hospitalar para os pacientes submetidos à cirurgia de redução da volume pulmonar para o enfisema era de 14 dias e que este prazo foi encurtado para oito dias após a introdução do uso da válvula de Heimlich conectada aos drenos torácicos. $\mathrm{O}$ uso da válvula de Heimlich é também psicologicamente mais aceitável por pacientes ope- 
rados propensos à ansiedade e depressão. 0 sistema estimula-os mentalmente, facilitando a deambulação, independência e alta hospitalar precoce ${ }^{(13)}$.

Pacientes com pneumotórax espontâneo recidivante e que haviam sido submetidos à drenagem tubular convencional sob selo d'água puderam comparar os dois métodos e informaram que a diferença foi notável. Nesta série destacamos outras vantagens do sistema de drenagem com a válvula de H eimlich: a válvula está disponível em embalagem estéril e descartável, o paciente também pode ser tratado ambulatorialmente e, finalmente, o sistema é mais fácil de ser compreendido pela equipe médica, de enfermagem e, fundamentalmente, pelos pacientes. Neste estudo pôde-se verificar a alta precoce dos pacientes e seu

\section{REFERÊNCIAS}

1. Heimlich HJ. Valve drainage of the pleural cavity. Chest 1968;53: 282-7.

2. Mercier C, Pagé A, Verdant A, Cossete R, Dontigny L, Pelletier LC. Outpatient management of intercostal tube drainage in spontaneous pneumothorax. Ann Thorac Surg 1976;22:163-5.

3. Clagett OT. The management of spontaneous pneumothorax. J Thorac Cardiovasc Surg 1968;55:761-2.

4. Campisi P, Voitk AJ . Outpatient treatment of spontaneous pneumothorax in a community hospital using a Heimlich valve: a case series. J Emerg Med 1997;15:115-9.

5. Playfair GE. Case of empyema treated by aspiration and subsequently by drainage: recovery. Br Med J 1875;1:45.

6 . Robinson S. Acute thoracic empyema. Avoidance of chronic empyema. Rib trephining for suction drainage. Boston Med Surg J 1910; 163:561-70.

7. Lilienthal $\mathrm{H}$. Thoracic surgery: the surgical treatment of thoracic disease. Philadelphia: Saunders, 1926: 1:54,52-6; 2:156-7.

8. Van Hengel $P$, Van de Bergh JHAM. Heimlich valve treatment and outpatient management of bilateral metastatic pneumothorax. Chest 1994;105:1586-7. acompanhamento ambulatorial. Além disso, em estudo anterior, pudemos demonstrar as vantagens econômicas e do melhor uso das facilidades hospitalares, sem detrimento na qualidade do atendimento oferecido ao paciente, quando tratado ambulatorialmente com esse sistema ${ }^{(14)}$.

Em conclusão, na série estudada, a válvula de H eimlich mostrou-se eficiente na resolução do pneumotórax de diferentes etiologias, bem como nas fístulas aéreas pós-operatórias. Sua manipulação foi mais simples e rápida do que a historicamente observada com os sistemas convencionais. Adicionalmente, a boa tolerância referida pela maioria absoluta $(94,8 \%)$ dos pacientes é fator que determina maior precocidade de alta hospitalar, bem como incentiva o tratamento ambulatorial do pneumotórax.

9. Trachiotis GD, Vricella LA, Alyono D, Aaron BL, Hix WR. Management of AIDS-related pneumothorax. Ann Thorac Surg 1996;62:160813.

10. Driver AG, Peden J G, Adams GA, Rumley RL. Heimlich valve treatment of Pneumocystis carinii associated pneumothorax. Chest 1991; $100: 281-2$.

11. Light RW, Hamm H. Pleural disease and acquired immune deficiency syndrome. Eur Respir J 1997;10:2638-43.

12. Robert J S, Bratton SL, Brogan TV. Efficacy and complications of percutaneous pigtail catheters for thoracostomy in pediatric patients. Chest 1998;114:1116-21.

13. McKenna RJ, Fischel R, Brenner M, Gelb AF. Use of the Heimlich valve to shorten hospital stay after lung reduction surgery for emphysema. Ann Thorac Surg 1996;61:1115-7.

14. Beyruti R, Fernandez A, Milanes J RC, Villiger LEO, Cipriano FG, Jatene FB. Nossa experiência com o uso da válvula de Heimlich no tratamento do pneumotórax. J Pneumol 1999;25(Suppl 1):2. 